PROCEEDINGS OF THE

AMERICAN MATHEMATICAL SOCIETY

Volume 140, Number 12, December 2012, Pages 4351-4357

S 0002-9939(2012)11284-5

Article electronically published on April 20, 2012

\title{
A NOTE ON THE UNIQUENESS OF SOLUTIONS FOR THE YAMABE PROBLEM
}

\author{
L. L. DE LIMA, P. PICCIONE, AND M. ZEDDA
}

(Communicated by Jianguo Cao)

\begin{abstract}
Using recent results on the compactness of the space of solutions of the Yamabe problem, we show that in conformal classes of metrics near the class of a nondegenerate solution which is unique (up to scaling) the Yamabe problem has a unique solution as well. This provides examples of a local extension, in the space of conformal classes, of a well-known uniqueness criterion due to Obata.
\end{abstract}

\section{INTRODUCTION}

A celebrated result obtained by a combined effort of Yamabe [19], Trudinger [18, Aubin [1] and Schoen [16 gives the existence of a constant scalar curvature metric in every conformal class of Riemannian metrics on a compact manifold $M$. Multiplicity results for solutions of the Yamabe problem have been proved in a variety of cases; see for instance [7, 15].

Recently, remarkable results on the noncompactness of the space of solutions for the Yamabe problem have been obtained, first by Brendle (see [4]), and subsequently by Brendle and Marques (see [5]). A compactness theorem for $n \leq 24$ was proved in [8] by Khuri, Marques and Schoen. Manuscript [6] is an interesting survey on the compactness and the noncompactness issues for the Yamabe problem.

In the present paper we address the question of uniqueness of solutions of the Yamabe problem in conformal classes. A well-known result by Obata 14 says that an Einstein metric, which happens to be a critical point of the Hilbert-Einstein functional in the manifold of all Riemannian metrics with total volume 1 , is the unique constant scalar curvature metric in its conformal class up to scaling, provided it is not conformal to a round metric on the sphere. Such metrics are nondegenerate global minima of the Hilbert-Einstein functional. The structure of the set of constant scalar curvature metrics in conformal classes near a nondegenerate constant scalar curvature metric has been studied in the smooth case by Koiso [10] (see also 2. Ch. 4]), using the language of ILH (inverse limit Hilbert) manifolds. In this paper we will establish that, near a nondegenerate solution of the Yamabe problem, the set of unit volume and constant scalar curvature metrics of class $\mathcal{C}^{k, \alpha}, k \geq 2$, on a given compact manifold $M$, with $m=\operatorname{dim}(M) \geq 3$, is a smooth embedded

Received by the editors February 11, 2011 and, in revised form, February 15, 2011 and June 2, 2011.

2010 Mathematics Subject Classification. Primary 53C25, 58E11.

The first author is partially sponsored by CNPq and Funcap, Brazil.

The second author is partially sponsored by CNPq and Fapesp, Brazil.

The third author is supported by RAS through a grant financed with the "Sardinia PO FSE 2007-2013" funds and provided according to the L.R. 7/2007. 
submanifold of the set of metrics, which is (strongly) transversal to the conformal classes (Proposition [3). This transversality property immediately yields a local uniqueness result for the Yamabe problem in conformal classes near to that of a nondegenerate solution (Corollary 4). The proof is based on an abstract formulation of the inverse mapping theorem in vector bundles, discussed in Section 2, whose proof only involves standard infinite dimensional differential geometry techniques, thus completely avoiding the ILH formalism. Moreover, the local uniqueness result, combined with the compactness results of [8, 11, [12 and [13, gives a global uniqueness result for solutions of the Yamabe problem in conformal classes near that of a nondegenerate constant scalar curvature metric which is the unique unit volume solution in its conformal class (Theorem 5). This applies in particular to certain families of Einstein metrics appearing in Obata's uniqueness result mentioned above. Examples include any space form $M$ (other than the round sphere) satisfying either $m \leq 7$ or $m \leq 24$ and $M$ is spin or any homogeneous Einstein manifold which is not locally conformally flat. Thus our result provides examples of a local extension, in the space of conformal classes, of Obata's uniqueness criterion. Observe also that our global uniqueness result generalizes a well-known result by Böhm, Wang and Ziller that near a unit volume Einstein metric which is not conformally equivalent to the round sphere all unit volume scalar curvature metrics must be Yamabe metrics, i.e., global minima of the Hilbert-Einstein functionals; see [3, Theorem 5.1].

\section{A FIBERWISE INVERSE FUNCTION THEOREM}

Let us first state an abstract formulation of an inverse mapping theorem which is suited to our purposes. Let us introduce the following notation, terminology and conventions. Given a Banach manifold $\mathcal{M}$, by a smooth Banach vector bundle $\mathcal{E}$ on $\mathcal{M}$ with typical fiber $E_{0}$ we always mean a vector bundle whose transition maps are smooth as maps from open subsets $U \subset \mathcal{M}$ to $\mathrm{GL}\left(E_{0}\right)$. The zero section of a vector bundle will be denoted by $\mathbf{0}$; given $x_{0} \in \mathcal{M}$, the zero of the fiber $\mathcal{E}_{x_{0}}$ is denoted by $\mathbf{0}_{x_{0}}$. If $s: \mathcal{M} \rightarrow \mathcal{E}$ is a smooth section of the vector bundle $\mathcal{E}$ and $s\left(x_{0}\right)=\mathbf{0}_{x_{0}}$, then the vertical derivative $\mathrm{d}^{\mathrm{ver}} s\left(x_{0}\right): T_{x_{0}} \mathcal{M} \rightarrow \mathcal{E}_{x_{0}}$ is the linear map defined as the composition $P_{\text {ver }} \circ \mathrm{d} s\left(x_{0}\right)$, where $P_{\text {ver }}: T_{\mathbf{0}_{x_{0}}} \mathcal{E} \rightarrow \mathcal{E}_{x_{0}}$ is the projection relative to the decomposition $T_{\mathbf{0}_{x_{0}}} \mathcal{E} \cong T_{\mathbf{0}_{x_{0}}} \mathbf{0} \oplus T_{\mathbf{0}_{x_{0}}} \mathcal{E}_{x_{0}} \cong T_{\mathbf{0}_{x_{0}}} \mathbf{0} \oplus \mathcal{E}_{x_{0}}$. By a smooth distribution on $\mathcal{M}$ we mean a smooth vector subbundle of $T \mathcal{M}$; a submanifold $\mathcal{L} \subset \mathcal{M}$ is said to be strongly transversal to a distribution $\mathcal{D} \subset T \mathcal{M}$ if at every point $x \in \mathcal{L}, T_{x} \mathcal{L}$ is a closed complement of $\mathcal{D}_{x}$, i.e., if $T_{x} \mathcal{L} \cap \mathcal{D}_{x}=\{0\}$ and $T_{x} \mathcal{L}+\mathcal{D}_{x}=T_{x} \mathcal{M}$.

1. Proposition. Let $\mathcal{M}$ be a Banach manifold, $\mathcal{D} \subset T \mathcal{M}$ be a smooth distribution on $\mathcal{M}, \mathcal{E} \rightarrow \mathcal{M}$ a smooth vector bundle and $\mathfrak{i}: \mathcal{E} \rightarrow \mathcal{D}^{*}$ an injective smooth vector bundle morphism. Assume that $F: \mathcal{M} \rightarrow \mathbb{R}$ is a smooth function and that $s$ is a smooth section of $\mathcal{E}$ satisfying

$$
\mathfrak{i}(s(x))=\left.\mathrm{d} F(x)\right|_{\mathcal{D}_{x}}, \quad \forall x \in \mathcal{M} .
$$

Assume that $\left.\mathrm{d} F\left(x_{0}\right)\right|_{\mathcal{D}_{x_{0}}}=0$ (or, equivalently, that $s\left(x_{0}\right)=0$ ) at some $x_{0} \in \mathcal{M}$, and that

$$
\left.\mathrm{d}^{\mathrm{ver}} s\left(x_{0}\right)\right|_{\mathcal{D}_{x_{0}}}: \mathcal{D}_{x_{0}} \longrightarrow \mathcal{E}_{x_{0}}
$$

is an isomorphism of Banach spaces. 
Then, there exists an open subset $U \subset \mathcal{M}$ containing $x_{0}$ such that the set

$$
\mathcal{L}=\left\{x \in U:\left.\mathrm{d} F(x)\right|_{\mathcal{D}_{x}}=0\right\}
$$

is a smooth embedded submanifold of $\mathcal{M}$ which is strongly transversal to $\mathcal{D}$.

Proof. The assumption that $\mathrm{d}^{\mathrm{ver}} s\left(x_{0}\right)$ is an isomorphism from $\mathcal{D}_{x_{0}}$ to $\mathcal{E}_{x_{0}}$ implies that $s$ is transversal to the zero section of $\mathcal{E}$ at $x_{0}$. Observe that the composition of $\mathrm{d} s\left(x_{0}\right): T_{x_{0}} \mathcal{M} \rightarrow T_{\mathbf{0}_{x_{0}}} \mathcal{E}$ with the projection $T_{\mathbf{0}_{x_{0}}} \mathcal{E} \rightarrow T_{\mathbf{0}_{x_{0}}} \mathcal{E} / T_{x_{0}} \mathbf{0} \cong \mathcal{E}_{x_{0}}$ is identified with the vertical derivative $\mathrm{d}^{\mathrm{ver}} s\left(x_{0}\right)$, which is therefore surjective. Moreover, the space $\mathcal{D}_{x_{0}}$ is a closed complement of $\operatorname{Ker}\left(\mathrm{d} s\left(x_{0}\right)\right)$; i.e., $s$ is strongly transversal to $\mathbf{0}$ at $x_{0}$. Since $\mathfrak{i}$ is injective, from (1) it follows easily that the set $\mathcal{L}$ can also be described as

$$
\mathcal{L}=U \cap s^{-1}(\mathbf{0})
$$

it follows that for $U$ sufficiently small, $\mathcal{L}$ is a smooth embedded submanifold of $\mathcal{M}$ and that for $x \in \mathcal{L}, T_{x} \mathcal{L}=\operatorname{Ker}(\mathrm{d} s(x))$. Note that for $x \in \mathcal{L}$ near $x_{0}, \mathcal{D}_{x}$ is a closed complement of $\operatorname{Ker}(\mathrm{d} s(x))$; i.e., $\mathcal{L}$ is strongly transversal to $\mathcal{D}$.

\section{The MANifold of Unit VOlume Metrics AND CONFORMAL ClASSES}

We will now discuss an application of Proposition 1 in the context of constant scalar curvature metrics. Let $M$ be a compact manifold, $m=\operatorname{dim}(M) \geq 3$, and given $k \geq 2, \alpha \in] 0,1]$, denote by $\operatorname{Met}^{k, \alpha}(M)$ the set of Riemannian metrics of class $\mathcal{C}^{k, \alpha}$ on $M$, i.e., the open subset of the Banach space $\Gamma^{k, \alpha}\left(\vee^{2} T M^{*}\right)$ consisting of all $\mathcal{C}^{k, \alpha}$-sections of the vector bundle of $\mathrm{V}^{2}\left(T M^{*}\right)$ consisting of symmetric $(0,2)$-tensors on $M$ that are pointwise positive definite. For $g \in \operatorname{Met}^{k, \alpha}(M)$, we will denote by $\nu_{g}$ the volume form (or density, if $M$ is not orientable) of $g$, by $\operatorname{Ric}_{g}$ the Ricci curvature of $g$, and by $\kappa_{g}$ its scalar curvature function, which is a function of class $\mathcal{C}^{k-2, \alpha}$ on $M$. The volume function $\mathcal{V}$ on $\operatorname{Met}^{k, \alpha}(M)$ is defined by

$$
\mathcal{V}(g)=\int_{M} \nu_{g}
$$

Observe that $\mathcal{V}$ is smooth, and its differential is given by

$$
\mathrm{d} \mathcal{V}(g) h=\frac{1}{2} \int_{M} \operatorname{tr}_{g}(h) \nu_{g}
$$

for all $h \in \Gamma^{k, \alpha}\left(\bigvee^{2} T M^{*}\right)$. Set

$$
\mathcal{M}=\left\{g \in \operatorname{Met}^{k, \alpha}(M): \mathcal{V}(g)=1\right\} ;
$$

this is a smooth embedded submanifold of $\operatorname{Met}^{k, \alpha}(M)$. For $g \in \mathcal{M}$, the tangent space $T_{g} \mathcal{M}$ is given by

$$
T_{g} \mathcal{M}=\left\{h \in \Gamma^{k, \alpha}\left(\vee^{2}\left(T M^{*}\right)\right): \int_{M} \operatorname{tr}_{g}(h) \nu_{g}=0\right\} .
$$

Consider the Hilbert-Einstein functional $F: \mathcal{M} \longrightarrow \mathbb{R}$, defined by

$$
F(g)=\int_{M} \kappa_{g} \nu_{g} ;
$$

this is a smooth function on $\mathcal{M}$, whose critical points are the Einstein metrics of volume 1 on $M$. Finally, for $g \in \mathcal{M}$, let $\mathcal{D}_{g}$ be the closed subspace of $T_{g} \mathcal{M}$ given by

$$
\mathcal{D}_{g}=\left\{\varphi \cdot g: \varphi \in \mathcal{C}^{k, \alpha}(M, \mathbb{R}): \int_{M} \varphi \nu_{g}=0\right\} .
$$


2. Lemma. As to the above setting, the following hold:

(a) $\mathcal{D}=\bigcup_{g \in \mathcal{M}} \mathcal{D}_{g}$ is a smooth integrable distribution on $\mathcal{M}$;

(b) for $g \in \mathcal{M}$, the maximal connected integral submanifold of $\mathcal{D}$ through $g$ is the subset $D_{g} \subset \mathcal{M}$ given by

$$
D_{g}=\left\{\phi \cdot g: \phi \in \mathcal{C}^{k, \alpha}(M, \mathbb{R}): \phi>0, \int_{M} \phi^{\frac{m}{2}} \nu_{g}=1\right\} .
$$

Proof. Statement (a) is proven by showing that $\mathcal{D}$ is the image of a smooth vector bundle morphism with constant rank 1 Consider the trivial vector bundles (over M) $E_{1}=\mathcal{M} \times \mathcal{C}^{k, \alpha}(M, \mathbb{R}), E_{2}=\mathcal{M} \times \mathbb{R}$, and the smooth vector bundle morphism $f_{1}: E_{1} \rightarrow E_{2}$ defined by

$$
f_{1}(g, \varphi)=\left(g, \int_{M} \varphi \nu_{g}\right) .
$$

Clearly, $f_{1}$ is surjective, and its kernel

$$
E_{3}=\bigcup_{g \in \mathcal{M}}\{g\} \times\left\{\varphi \in \mathcal{C}^{k, \alpha}(M, \mathbb{R}): \int_{M} \varphi \nu_{g}=0\right\},
$$

which is closed and complemented, is a smooth vector subbundle of $E_{1}$. Now, the smooth bundle morphism $f_{2}: E_{3} \rightarrow T \mathcal{M}$ defined by $(g, \varphi) \mapsto(g, \varphi \cdot g)$ has image $\mathcal{D}$, and it has constant rank. Namely, the fiber bundle morphism $T \mathcal{M} \ni(g, \bar{g}) \mapsto$ $\left(g, \frac{1}{m} \operatorname{tr}_{g}(\bar{g})\right) \in E_{3}$ is a left inverse of $f_{2}$. This proves (a). For (b), observe that $\phi^{\frac{m}{2}} \nu_{g}$ is the volume form of the metric $\phi \cdot g$. For $g \in \mathcal{M}$, consider the smooth map $\mathcal{V}_{g}: \mathcal{C}^{k, \alpha}(M, \mathbb{R}) \ni \phi \mapsto \int_{M} \phi^{\frac{m}{2}} \nu_{g} \in \mathbb{R}$, whose derivative is

$$
\mathrm{d} \mathcal{V}_{g}(\phi) \varphi=\frac{m}{2} \int_{M} \phi^{\frac{m}{2}-1} \varphi \nu_{g}
$$

It is easily checked that $\mathcal{V}_{g}$ has no critical values, so that the set $\mathcal{V}_{g}^{-1}(1)$ is a smooth embedded submanifold of $\mathcal{C}^{k, \alpha}(M, \mathbb{R})$. Now, for $g \in \mathcal{M}$, the map $I: \mathcal{V}_{g}^{-1}(1) \ni \phi \mapsto$ $\phi \cdot g \in \mathcal{M}$ is smooth, and a smooth left inverse is given by the map $\bar{g} \mapsto \frac{1}{m} \operatorname{tr}_{g}(\bar{g})$. It follows that $I$ is an embedding, and its image is $D_{g}$, which is therefore a smooth embedded submanifold of $\mathcal{M}$. Using (5) at $\phi=1$, one proves easily $T_{g} D_{g}=\mathcal{D}_{g}$; i.e., $D_{g}$ is an integral submanifold of $\mathcal{D}$. It is easy to see that $D_{g}$ is connected (it is homeomorphic to the set of positive functions $\phi \in \mathcal{C}^{k, \alpha}$ satisfying $\int_{M} \phi^{\frac{m}{2}} \nu_{g}=1$ )

\footnotetext{
${ }^{1}$ Given vector bundles $\mathcal{E}$ and $\mathcal{F}$ over a manifold $M$, with typical fibers $E_{0}$ and $F_{0}$ respectively, a vector bundle morphism $\phi: \mathcal{E} \rightarrow \mathcal{F}$ is said to have constant rank if, identifying the fiber $\mathcal{E}_{x} \cong E_{0}$, $\mathcal{F}_{x} \cong F_{0}, x \in U \subset M$, via local trivializations of $\mathcal{E}$ and $\mathcal{F}$, there exists a closed subspace $G \subset F_{0}$ such that $\phi_{x}\left(E_{0}\right) \oplus G=F_{0}$ for all $x \in U$. Such a condition does not depend on the choice of trivializations. If $\phi$ has constant rank, then $\phi(\mathcal{E})$ is a subbundle of $\mathcal{F}$.
}

Lemma. If $\phi: \mathcal{E} \rightarrow \mathcal{F}$ has a left inverse, then it has a fiberwise closed image and constant rank.

Proof. A bounded linear operator $T: E_{0} \rightarrow F_{0}$ between Banach spaces that admits a left inverse $S: F_{0} \rightarrow E_{0}$ has a closed image, and $\operatorname{Ker}(S)$ is a closed complement for $\operatorname{Im}(T)$ (trivial). Given trivializations of $\mathcal{E}$ and $\mathcal{F}$ over an open subset $U \subset M$, write $\phi$ and its left inverse $\psi$ as maps $U \ni x \mapsto \phi_{x} \in \operatorname{Lin}\left(E_{0}, F_{0}\right)$ and $U \ni x \mapsto \psi_{x} \in \operatorname{Lin}\left(F_{0}, E_{0}\right)$. Now, given $x_{0} \in U$, for all $x \in U$ the map $\left(\psi_{x_{0}} \circ \phi_{x}\right)^{-1} \circ \psi_{x_{0}}$ is a left inverse of $\phi_{x}$. It follows that

$$
\operatorname{Ker}\left(\left(\psi_{x_{0}} \circ \phi_{x}\right)^{-1} \circ \psi_{x_{0}}\right)=\operatorname{Ker}\left(\psi_{x_{0}}\right)
$$

is a fixed closed complement of $\operatorname{Im}\left(\phi_{x}\right)$ for all $x \in U$; i.e., $\phi$ has constant rank. 
and that $\mathcal{M}$ is the disjoint union of all $D_{g}$ 's. It follow 2 that every $D_{g}$ is a maximal connected integral submanifold of $\mathcal{D}$.

The $\mathcal{C}^{k, \alpha}$-conformal class of $g$ is the submanifold of $\operatorname{Met}^{k, \alpha}(M)$,

$$
[g]_{k, \alpha}=\left\{\phi \cdot g: \phi \in \mathcal{C}^{k, \alpha}(M, \mathbb{R}): \phi>0\right\} ;
$$

clearly, $D_{g}=\mathcal{M} \cap[g]_{k, \alpha}$. From the proof of Lemma 2, part (b), one deduces easily that $\mathcal{M}$ and $[g]_{k, \alpha}$ are transversal.

Let us recall the first and the second variational formula for the function $F$ restricted to a conformal class $D_{g}$; see [9, 17] for details. We identify the tangent space $T_{g} D_{g}$ with the space of functions $f \in \mathcal{C}^{k, \alpha}(M, \mathbb{R})$ with $\int_{M} f \nu_{g}=0$. Given one such $f$, the first derivative of $\left.F\right|_{D_{g}}$ in the direction $f$ is

$$
\mathrm{d} F(g)(f)=\frac{m-2}{2} \int_{M} f \kappa_{g} \nu_{g}
$$

if $g$ has constant scalar curvature, then

$$
\mathrm{d}^{2} F(g)(f, f)=\frac{m-2}{2} \int_{M}\left((m-1) \Delta_{g} f-\kappa_{g} f\right) f \nu_{g},
$$

where $\Delta_{g}$ is the Laplace-Beltrami operator of the metric $g$.

\section{The manifold of unit volume CONSTANT SCALAR CURVATURE METRICS}

Given $g \in \mathcal{M}$ with constant scalar curvature $\kappa_{g}$ in $M$, we say that $g$ is nondegenerate if either $\kappa_{g}=0$ or if $\frac{\kappa_{g}}{m-1}$ is not an eigenvalue of the Laplace-Beltrami operator $\Delta_{g}$ of $g$.

3. Proposition. Let $g_{*} \in \mathcal{M}$ be a nondegenerate constant scalar curvature metric. Then, there exists an open neighborhood $U$ of $g_{*}$ in $\mathcal{M}$ such that the set

$$
\left\{g \in U: \kappa_{g} \text { is constant }\right\}
$$

is a smooth embedded submanifold of $\mathcal{M}$ which is strongly transversal to the conformal classes.

Proof. This is a direct application of Proposition 1 to the following setup. The manifold $\mathcal{M}$ is defined in (3) and the distribution $\mathcal{D}$ in Lemma 2, The vector bundle $\mathcal{E}$ has fibers:

$$
\mathcal{E}_{g}=\left\{f \in \mathcal{C}^{k-2, \alpha}(M, \mathbb{R}): \int_{M} f \nu_{g}=0\right\}, \quad g \in \mathcal{M},
$$

and the vector bundle morphism $\mathfrak{i}: \mathcal{E} \rightarrow \mathcal{D}^{*}$ is induced by the $L^{2}$-pairing, i.e.,

$$
\mathfrak{i}_{g}\left(f_{1}\right) f_{2}=\int_{M} f_{1} f_{2} \nu_{g}, \quad g \in \mathcal{M}, f_{1} \in \mathcal{E}_{g}, f_{2} \in \mathcal{D}_{g} .
$$

\footnotetext{
${ }^{2}$ We are using the following elementary result:
}

Lemma. Let $\mathcal{M}$ be a manifold and $\mathcal{D} \subset T \mathcal{M}$ an integrable distribution. Assume that $\mathcal{M}$ is the disjoint union $\bigcup_{i \in I} S_{i}$ of connected integral submanifolds of $\mathcal{D}$. Then, every $S_{i}$ is a maximal connected integral submanifold of $\mathcal{D}$. 
The function $F: \mathcal{M} \rightarrow \mathbb{R}$ is the Hilbert-Einstein functional (4), and the section $s: \mathcal{M} \rightarrow \mathcal{E}$ is given by

$$
\begin{aligned}
s(g) & =-\operatorname{tr}_{g}\left(\operatorname{Ric}_{g}-\frac{1}{2} g \kappa_{g}\right)+\int_{M}\left(\operatorname{Ric}_{g}-\frac{1}{2} g \kappa_{g}\right) \nu_{g} \\
& =\left(\frac{m}{2}-1\right) \kappa_{g}-\left(\frac{m}{2}-1\right) \int_{M} \kappa_{g} \nu_{g} .
\end{aligned}
$$

Note that $s(g)=0$ if and only if $\kappa_{g}$ is constant. Given one such $g$, the composition $\left.\mathfrak{i}_{g} \circ \mathrm{d}^{\text {ver }} s(g)\right|_{\mathcal{D}_{g}}: \mathcal{D}_{g} \rightarrow \mathcal{D}_{g}^{*}$ is identified with the second variation $\mathrm{d}^{2}\left(\left.F\right|_{D_{g}}\right)(g)$ of the restriction of $F$ to the integral submanifold $D_{g}$. Using (66) one checks easily that equality (1) in Proposition 11 holds. Moreover, using formula (7), the map $\left.\mathrm{d}^{\mathrm{ver}} s\left(g_{*}\right)\right|_{\mathcal{D}_{g_{*}}}: \mathcal{D}_{g_{*}} \rightarrow \mathcal{E}_{g_{*}}$ is identified with the linear operator

$$
f \longmapsto \frac{m-2}{2}\left((m-1) \Delta_{g_{*}} f-\kappa_{g_{*}} f\right),
$$

defined on the space of functions $f \in \mathcal{C}^{k, \alpha}(M, \mathbb{R})$ with $\int_{M} f \nu_{g_{*}}=0$ and taking values in the space of functions $f \in \mathcal{C}^{k-2, \alpha}(M, \mathbb{R})$ with $\int_{M} f \nu_{g_{*}}=0$. Observe that such an operator preserves the space of functions with zero integral. The assumption of nondegeneracy for $g_{*}$ is equivalent to the fact that such an operator is an isomorphism, and Proposition 1 can be applied to obtain the conclusion.

4. Corollary. Let $g_{*} \in \mathcal{M}$ be a nondegenerate constant scalar curvature metric. Then, there exists an open neighborhood $U$ of $g_{*}$ in $\mathcal{M}$ such that every conformal class of metrics in $M$ contains at most one unit volume constant curvature metric in $U$.

Proof. Strong transversality of the manifold (8) with the conformal classes implies local uniqueness of intersections.

We are ready for our main result, which gives a global uniqueness result for the Yamabe problem.

5. Theorem. Assume that $g_{*} \in \mathcal{M}$ is a nondegenerate constant scalar curvature metric on $M$, which is the unique unit volume constant scalar curvature metric in its conformal class. Assume in addition that either one of the following conditions is satisfied:

(a) $\operatorname{dim}(M) \leq 7$;

(b) the Weyl tensor $W_{g_{*}}$ of $g_{*}$ satisfies

$$
\left|W_{g_{*}}(p)\right|+\left|\nabla W_{g_{*}}(p)\right|>0, \quad \forall p \in M ;
$$

(c) $\operatorname{dim}(M) \leq 24$ and $M$ is spin.

Then, for $g$ sufficiently $\mathcal{C}^{2, \alpha}$-close to $g_{*}$, there is a unique unit volume constant scalar curvature metric in the conformal class of $g$.

Proof. Either one of conditions (a), (b) or (c) guarantees that, given a sequence $g_{n}$ of Riemannian metrics on $M$ with $\lim _{n \rightarrow \infty} g_{n}=g_{*}$, the set of unit volume constant scalar curvature metrics that are conformal to some $g_{n}$ is compact. This follows from the arguments in [8, 11, 13, see in particular [8, Lemma 10.1]. Note that condition (9) is open (in the $\mathcal{C}^{1}$-topology); hence it holds for every $g_{n}$ with $n$ sufficiently large if it holds for $g_{*}$. By contradiction, assume the existence of a sequence $g_{n}$ of unit volume constant scalar curvature metrics on $M$, with $\lim _{n \rightarrow \infty} g_{n}=g_{*}$, and such that 
the conformal class of $g_{n}$ contains a distinct unit volume constant scalar curvature metric $h_{n} \neq g_{n}$ for all $n$. By compactness, up to subsequences there exists the limit $\lim _{n \rightarrow \infty} h_{n}=h_{*}$; by continuity, $h_{*}$ is a unit volume constant scalar curvature metric in the conformal class of $g_{*}$. By uniqueness in the conformal class of $g_{*}$, it must be $h_{*}=g_{*}$, but this contradicts the local uniqueness result of Corollary 4, which concludes the proof.

\section{REFERENCES}

[1] T. Aubin, Équations différentielles non-linéaires et problème de Yamabe concernant la courbure scalaire, J. Math. Pures Appl. 55 (1976), 269-296. MR0431287 (55:4288)

[2] A. L. Besse, Einstein manifolds, reprint of the 1987 edition. Classics in Mathematics, Springer-Verlag, Berlin, 2008. MR,2371700 (2008k:53084)

[3] C. Böhm, M. Wang, W. Ziller, A variational approach for compact homogeneous Einstein manifolds, Geom. Funct. Anal. 14 (2004), no. 4, 681-733. MR2084976 (2005g:53074)

[4] S. Brendle, Blow-up phenomena for the Yamabe equation, J. Amer. Math. Soc. 21 (2008), no. 4, 951-979. MR2425176 (2009m:53084)

[5] S. Brendle, F. C. Marques, Blow-up phenomena for the Yamabe equation. II, J. Differential Geom. 81 (2009), no. 2, 225-250. MR2472174 (2010k:53050)

[6] S. Brendle, F. C. Marques, Recent progress on the Yamabe problem, preprint, arXiv:1010.4960v1.

[7] L. L. de Lima, P. Piccione, M. Zedda, On bifurcation of solutions of the Yamabe problem in product manifolds, preprint, arXiv:1012.1497v1.

[8] M. A. Khuri, F. C. Marques, R. M. Schoen, A compactness theorem for the Yamabe problem, J. Differential Geom. 81 (2009), no. 1, 143-196. MR2477893 (2010e:53065)

[9] N. Koiso, On the second derivative of the total scalar curvature, Osaka J. Math. 16 (1979), 413-421. MR539596 (80j:53047)

[10] N. Koiso, A decomposition of the space $\mathcal{M}$ of Riemannian metrics on a manifold, Osaka J. Math. 16 (1979), 423-429. MR539597 (80g:53034)

[11] Y. Y. Li, L. Zhang, Compactness of solutions to the Yamabe problem. II, Calc. Var. Partial Differential Equations 24 (2005), no. 2, 185-237. MR2164927 (2006f:53049)

[12] Y. Y. Li, L. Zhang, Compactness of solutions to the Yamabe problem. III, J. Funct. Anal. 245 (2007), 438-474. MR 2309836 (2008f:53038)

[13] F. C. Marques, A priori estimates for the Yamabe problem in the non-locally conformally flat case, J. Differential Geom. 71 (2005), no. 2, 315-346. MR2197144 (2006i:53046)

[14] M. Obata, The conjectures on conformal transformations of Riemannian manifolds, J. Differential Geometry 6 (1971/72), 247-258. MR0303464(46:2601)

[15] J. Petean, Metrics of constant scalar curvature conformal to Riemannian products, Proc. Amer. Math. Soc. 138 (2010), no. 8, 2897-2905. MR.2644902

[16] R. Schoen, Conformal deformation of a Riemannian metric to constant scalar curvature, J. Diff. Geom. 20 (1984), 479-495. MR788292 (86i:58137)

[17] R. Schoen, Variational theory for the total scalar curvature functional for Riemannian metrics and related topics, Topics in Calculus of Variations, Lecture Notes in Mathematics, 1365, Springer, Berlin, 1989, 120-154. MR994021 (90g:58023)

[18] N. Trudinger, Remarks concerning the conformal deformation of Riemannian structures on compact manifolds, Annali Scuola Norm. Sup. Pisa 22 (1968), 265-274. MR0240748 (39:2093)

[19] H. Yamabe, On a deformation of Riemannian structures on compact manifolds, Osaka J. Math. 12 (1960), 21-37. MR0125546 (23:A2847)

Departamento de Matemática, Universidade Federal do Ceará, Fortaleza, 60455-760, BRAZIL

Departamento de Matemática, Universidade de São Paulo, São Paulo, 05508-090, BRAZIL

Università degli Studi di Cagliari, Via Università 240, 09124 Cagliari, Italy

Current address: Departamento de Matemática, Universidade de São Paulo, São Paulo, 05508090, Brazil 